\section{BMJ Open} Ophthalmology

\title{
Establishing the influence of case complexity on the order of cataract lists: a cross-sectional survey
}

\author{
Keri McLean (D) , ${ }^{1}$ Mariantonia Ferrara, ${ }^{2}$ Rebecca Kaye, ${ }^{3}$ Vito Romano, ${ }^{1}$ \\ Stephen Kaye ${ }^{1}$
}

To cite: McLean $\mathrm{K}$, Ferrara M, Kaye R, et al. Establishing the influence of case complexity on the order of cataract lists: a cross-sectional survey. BMJ Open Ophthalmology 2021;6:e000809. doi:10.1136/ bmjophth-2021-000809

- Additional supplemental material is published online only. To view, please visit the journal online (http://dx.doi. org/10.1136/bmjophth-2021000809).

Received 25 May 2021 Accepted 31 August 2021
Check for updates

C Author(s) (or their employer(s)) 2021. Re-use permitted under CC BY-NC. No commercial re-use. See rights and permissions. Published by BMJ.

${ }^{1}$ Department of Eye and Vision Science, University of Liverpool Faculty of Health and Life Sciences, Liverpool, UK ${ }^{2}$ Newcastle Eye Centre, Royal Victoria Infirmary, Newcastle upon Tyne, UK

${ }^{3}$ Clinical and Experimental Sciences, Vision Sciences Group, University of Southampton Faculty of Medicine,

Southampton, UK

Correspondence to Dr Keri McLean; K.McLean@ liverpool.ac.uk

\section{ABSTRACT}

Objective Order of the theatre list and complexity of the cases are important considerations which are known to influence surgical outcomes. This survey aimed to establish their influence on cataract surgery.

Methods and Analysis Cataract surgeons ordered five cataract cases according to their surgical preference, first using case notes and second using composite ORs (CORs) for posterior capsule rupture. Descriptive and nonparametric statistics were used to analyse the data.

Results Between 11 June and 14 July 2020, 192 cataract surgeons from 14 countries completed the online survey. Majority of the surgeons (142 vs 50 ) preferred to choose the order of their list $(p<0.01)$ and to review the case notes prior to the day of surgery ( 89 vs $53 ; p=0.04$ ). $39.86 \%$ preferred to start with the less risky case and $32.43 \%$ reserved the last position on the list for the riskiest case. There was a significant trend to order the list in an ascending level of risk, independent of whether case notes or CORs were used. Additionally, $44.79 \%$ of the respondents indicated they would be happy to have their list order planned by an automated program based on their preferred risk score.

Conclusion This survey demonstrates that cataract surgeons prefer to choose the order of their theatre list and that the order is dependent on the complexity of cases. There is support among surgeons for automated list ordering based on an objective score for risk stratification, such as a COR.

\section{INTRODUCTION}

Cataract surgery, the most common ophthalmic surgical procedure, is both highly successful and generally safe; however, complications can arise. The most frequent, sight-threatening intraoperative complication is rupture of the posterior capsule of the lens (PCR), with or without vitreous loss. ${ }^{2}$ Management of PCR involves additional surgical steps and procedures and multiple follow-up visits and has a higher rate of postoperative complications, potentially resulting in worse visual outcomes. ${ }^{3}$ The preoperative quantification of PCR risk, therefore, is an important consideration for appropriate surgical planning. Narendran et $a l^{2}$ analysed 55567 cataract operations from

\section{Key messages}

What was already known about the subject?

> Current literature suggests placing complex cases at the end of the list may benefit outcomes as the surgeon's dexterity and comfort may be more optimal; however, it is unknown if cataract surgeons prefer to do this in practice or if case complexity influences how they prefer to order their theatre list.

What are the new findings?

> This study objectively demonstrates that cataract surgeons do prefer to choose the order of their theatre list and that their preference is dependent on the complexity of cases.

How might these results change the focus of research or clinical practice?

This study demonstrates that there is support among surgeons for automated list ordering based on an objective score for risk stratification, such as a composite $\mathrm{OR}$, to aid planning surgical lists and patient flow.

the National Cataract Database and developed a quantitative risk stratification method for PCR. In particular, the authors calculated the adjusted OR (AOR) for each relevant risk factor associated with PCR (table 1) and then provided composite OR (COR) as the product of the relevant AOR for a given case. ${ }^{2}$ The resulting COR was proposed as an indicator of the cumulative risk of PCR and indirectly the complexity of the particular case. ${ }^{2}$

Ruan et $a l^{4}$ demonstrated that the patient's characteristics (and thus case complexity) influence the psychological comfort of both the ophthalmic surgeon and the surgical team, which in turn impacts surgical performance. The authors concluded that the optimisation of the order of a cataract list would be beneficial for surgical performance and suggested placing the more complex cases at the end of the list, on the basis that the level of a surgeon's dexterity and comfort would be optimal. ${ }^{4}$ In this regard, CORs could 


\begin{tabular}{|c|c|c|}
\hline \multicolumn{2}{|c|}{ Risk factor for posterior capsule rupture } & \multirow{2}{*}{$\begin{array}{l}\text { Adjusted OR } \\
1.0\end{array}$} \\
\hline Age (years) & $<60$ & \\
\hline & $60-69$ & 1.14 \\
\hline & $70-79$ & 1.42 \\
\hline & $80-89$ & 1.58 \\
\hline & $90+$ & 2.37 \\
\hline \multirow[t]{2}{*}{ Gender } & Female & 1.00 \\
\hline & Male & 1.28 \\
\hline \multicolumn{2}{|l|}{ Glaucoma } & 1.30 \\
\hline \multicolumn{2}{|c|}{ Diabetic retinopathy } & 1.63 \\
\hline \multicolumn{2}{|c|}{ Brunescent/white cataract } & 2.99 \\
\hline \multicolumn{2}{|c|}{ No fundal view/vitreous opacities } & 2.46 \\
\hline \multicolumn{2}{|c|}{ Pseudoexfoliation/phacodonesis } & 2.92 \\
\hline \multirow[t]{3}{*}{ Pupil size } & Large & 1 \\
\hline & Medium & 1.14 \\
\hline & Small & 1.45 \\
\hline \multirow{2}{*}{$\begin{array}{l}\text { Axial length } \\
(\mathrm{mm})\end{array}$} & $<26.0$ & 1 \\
\hline & $\geq 26.0$ & 1.47 \\
\hline \multicolumn{2}{|c|}{ Doxazosin (alpha-blocker) } & 1.51 \\
\hline \multicolumn{2}{|c|}{ Not able to lie flat } & 1.27 \\
\hline \multirow[t]{6}{*}{ Surgeon grade } & Consultant & 1.0 \\
\hline & Associate specialist & 0.87 \\
\hline & Staff grade & 0.36 \\
\hline & Fellow & 1.65 \\
\hline & $\begin{array}{l}\text { Specialist Trainee Year 3-7 } \\
\text { (ST3-7) }\end{array}$ & 1.60 \\
\hline & $\begin{array}{l}\text { Specialist Trainee Year 1-2 } \\
\text { (ST1-2) }\end{array}$ & 3.73 \\
\hline
\end{tabular}

Specialty trainee refers to speciality registrars and residents. The number of years in ophthalmology training is specified.

be used as an objective measure to order the cataract list according to case complexity.

Furthermore, it may be argued that establishing a standardised process for the order of a list according to the surgeon's preference may optimise patient workflow on the day of surgery, which is imperative during the current COVID-19 pandemic.

In order to determine whether list order is an important surgical consideration and to establish if case complexity influences this, we surveyed cataract surgeons of different grades to evaluate their preference in ordering cataract lists.

\section{METHODS}

We used SurveyMonkey (1999-2020 SurveyMonkey) to create an anonymous web survey that was distributed between 11 June and 14 July 2020 to ophthalmology
Table 2 Five hypothetical scenarios, with corresponding composite ORs, scheduled for right eye phacoemulsification with intraocular lens implantation

Hypothetical case

Composite OR

A Woman in her late 70s, nuclear cataract. 1.84

PMH: glaucoma.

Medication: latanoprost 1 drop BE ON.

Axial length: $23.68 \mathrm{~mm}$.

Pupil size: large.

B Woman in her late 50s, nuclear cataract. 1.0

$\mathrm{PMH}$ : no other ophthalmic or medical

history.

Medication: nil regular.

Axial length: $24.25 \mathrm{~mm}$.

Pupil size: large.

C Man in his late 60s, nuclear cataract. $\quad 7.14$

PMH: pseudoexfoliation syndrome,

hypertension.

Medication: amlodipine $5 \mathrm{mg} \mathrm{OD,}$

simvastatin $40 \mathrm{mg}$ ON.

Axial length: $26.1 \mathrm{~mm}$.

Pupil size: medium.

D Man in his early 90s, brunescent

42.79

cataract, no fundal view. Due to heart

disease he is unable to lie flat.

$\mathrm{PMH}$ : hypertension, benign prostatic

hypertrophy, mild left ventricular failure.

Medications: atorvastatin $40 \mathrm{mg} \mathrm{ON}$,

bisoprolol $7.5 \mathrm{mg} \mathrm{OD}$, doxazosin

MR $8 \mathrm{mg} O D$, ramipril $2.5 \mathrm{mg} \mathrm{OD}$,

furosemide $40 \mathrm{mg} O D$.

Axial length: $23.72 \mathrm{~mm}$.

Pupil size: large.

E Man in his early 80s, nuclear cataract. 2.63

$\mathrm{PMH}$ : glaucoma.

Medication: Lumigan 1 drop BE ON.

Axial length: $20.8 \mathrm{~mm}$.

Pupil size: large.

BE, Both eyes; MR, Modified Release; OD, Once a day; ON, Once at night; $\mathrm{PMH}$, Past Medical History.

residents, fellows and specialists. All respondents consented to the use of the data collected for scientific purposes and publication.

The first section of the survey collected information on the demographic findings of the respondents, including country of practice and grade of surgeon (based on the risk factors for PCR; see table 1). The respondents were asked to specify if they prefer to choose to order their cataract list and, if so, based on what parameters (clinical notes or clinical examination on the day of surgery).

Respondents were then given the AORs for risk factors associated with PCR, as per the National Cataract Database (table 1), and advised how to calculate the COR for a given case as the product of the individual AORs. ${ }^{2}$ The higher the COR, the greater the risk of PCR. They were then presented with five hypothetical patients scheduled for right phacoemulsification with intraocular lens implantation. These cases were presented in 
the first question in terms of clinical findings, and in the following question in terms of the COR corresponding to those clinical findings (table 2). In both questions, respondents were asked to order the patients for a hypothetical theatre list, based only on their preference, that is, without any consideration to allergies, diabetic protocols or other theatre pressures. Respondents were advised to skip the question in the absence of any preference on the list order. This was accounted for during data analysis.

Finally, respondents were asked to declare if they would prefer the COR or the patient's clinical details as the main criterion to order the theatre list and, in cases where the patient's PCR risk was scored in clinic, would like to have the list automatically planned according to the patient's risk score and their known risk order preference.

Patients or the public were not involved in the design, or conduct, or reporting or dissemination plans of our research.

\section{Statistical analysis}

Categorical variables were summarised by percentages. Further statistical analyses were performed using GraphPad Prism V.6.01 for Windows (GraphPad Software, La Jolla, California, USA). The statistically significant difference between categorical variables was assessed by $\mathrm{X}^{2}$ test of independence or Fisher's exact test with Bonferroni correction when sample size was small. We considered a $\mathrm{p}$ value of $<0.05$ to be statistically significant except when Bonferroni correction had been applied to multiple comparisons. In these circumstances a $p$ value of $<0.005$ was considered statistically significant.

Spearman's rank correlations were also used to assess if there were significant correlations between the default order the case notes were presented (case A, case B, case $\mathrm{C}$, case $\mathrm{D}$, case $\mathrm{E}$ ), the default order the CORs were presented (OR 1.84, OR 2.63, OR 42.79, OR 7.14, OR 1.00 ) and the ascending order of CORs (OR 1.00, OR 1.84, OR 2.63, OR 7.14, OR 42.79) compared with the orders ranked by the participants.

\section{RESULTS}

Responses were received from 192 cataract surgeons of different grades and from 14 different countries, as summarised in table 3. Majority of the respondents declared that they would prefer to decide on the order of their operating list (142 vs 50; $p<0.0001$ ), regardless of grade or country of practice $(\mathrm{p}=0.35$ and $\mathrm{p}=0.36$, respectively). Of the 142 surgeons who preferred to choose their list order, the favourite modality of choice was based on a review of 'case-notes' (89 vs 53; $\mathrm{p}=0.04$ ), regardless of surgeon grade $(p=0.06)$. Approximately half of the respondents $(86 ; 44.79 \%)$ indicated they would be happy to have their list order planned by a computer program based on their preferred risk score, $60(31.25 \%)$ stated they preferred to order the list themselves and 46 $(23.96 \%)$ indicated they did not have preference.

Table 4 shows the responses regarding the preferred order of the five hypothetical scenarios based on case note review and COR. Of the 148 surgeons who ordered the five cases, $59(39.86 \%)$ preferred to start the list with case $\mathrm{B}(\mathrm{COR}=1.00)(\mathrm{p}<0.0001)$. Moreover, less risky cases (namely, case A or case B) were preferentially placed at the beginning of the list (see table 4 and online supplemental data 1 and 2). There was a tendency to place the intermediate cases (case $\mathrm{C}$ and case $\mathrm{E}$ ) either third or fourth on the list, whereas no preference was found for the second place on the list $(\mathrm{p}=0.12)$. The last position on the list was more commonly reserved for the riskiest case (case D) $(48 ; 32.43 \%)$, followed by the least risky case (case B) $(41 ; 27.7 \%)$ (see table 4 and online supplemental data 1 and 2). There was no significant correlation between the default order the case notes were presented (case A, case B, case C, case D, case E) and the orders ranked by the 148 participants who answered this question $(\mathrm{p}=0.0833)$.

When provided with the CORs only, 126 surgeons answered. There was a tendency to rank the cases according to the ascending COR (see table 4 and online supplemental data 1 and 2). The least risky case (case B) was more frequently chosen as first and the most difficult (case D) as last. When asked which of the two proposed

Table 3 Demographics of respondents by country and grade of surgeon

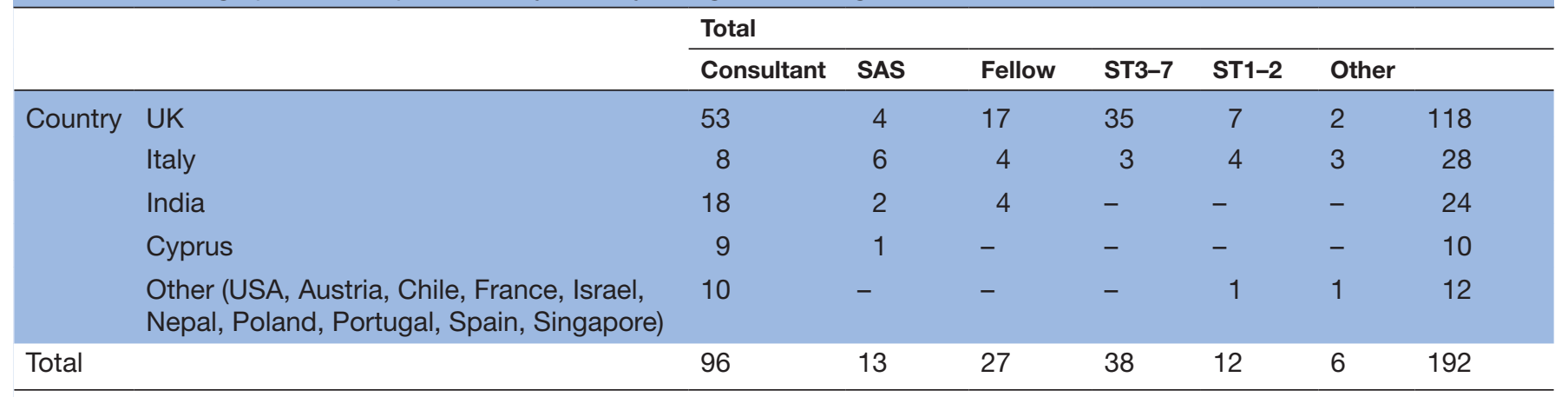

SAS, staff and associate specialist; ST1-2, Specialty trainee or resident with one to two years training in ophthalmology; ST3-7, Specialty trainee or resident with three to seven years training in ophthalmology. 


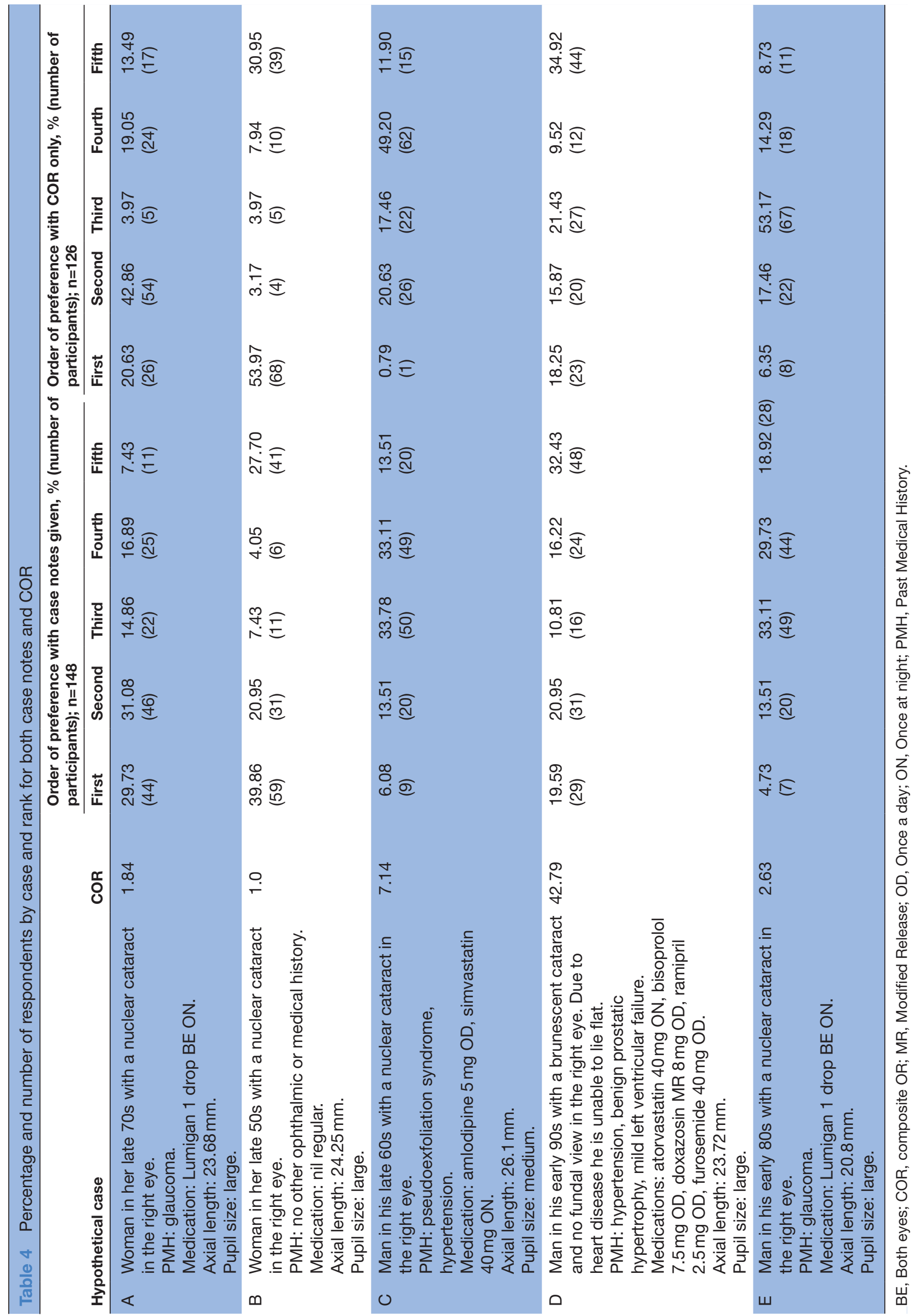


methods surgeons preferred to order the list, more respondents preferred case notes compared with CORs for risk of PCR (136 vs 11; $<<0.0001$ ), with no difference associated with the grade of surgeon $(\mathrm{p}=0.47)$. There was no significant correlation between orders ranked by the 126 participants, and either the default order in which the CORs were presented (OR 1.84, OR 2.63, OR 42.79, OR 7.14, OR 1.00) or the ascending order of the CORs (OR 1.00, OR 1.84, OR 2.63, OR 7.14, OR 42.79) ( $\mathrm{p}>0.999$ and $\mathrm{p}=0.083$, respectively).

Overall, whether given case details or CORs only, there was an evident tendency for cataract surgeons to order their list based on case complexity or risk of PCR, starting with the least risky and progressing to the most risky case (see table 4 and online supplemental data 1 and 2).

\section{DISCUSSION}

Several recent studies and reviews report that the order of the theatre list influences surgical performance. ${ }^{5-7}$ This includes a significant effect on the operating time, especially on lists where the same procedure is repeated. ${ }^{5}$ It has also been suggested that the complexity of the case could be a crucial criterion for list ordering. We, therefore, investigated how case complexity influences the order of the list in this survey-based study completed by varying grades of cataract surgeons. Hypothetical cases were used in this survey to negate confounding factors which may also influence theatre list order, such as theatre delays or over-runs, patient mobility and transport, patient comorbidities, general anaesthetic induction, special intraocular lenses, or bilateral sequential surgery.

We found that, regardless of grade and country of practice, most respondents preferred to choose the order of their cataract list and that the review of the case notes prior to the day of surgery was the current favourite modality of choice. This is congruent with the need to optimise theatre efficiency on the day of surgery. Indeed, reviewing patients' characteristics would allow the surgeon to plan in advance what equipment and settings she/he may need (such as a different position, pupil expander, capsule dye, capsular tension rings, etc), helping to facilitate a prompt start on the day of surgery. The importance of this prompt start is highlighted by the Royal College of Anaesthetists, who advocate starting the list within $15 \mathrm{~min}$ of the scheduled start time as one of the three main recommendations for day surgery, including ophthalmic surgery. ${ }^{8}$

Moreover, preplanning the list order with case complexity in mind may offer at least two additional advantages which are particularly relevant during the current COVID-19 pandemic. First, a known and reliable list order could allow the ward staff to more efficiently optimise patient flow to comply with the enhanced COVID-19 infection prevention and control measures, such as staggering patient arrival times to minimise the number of patients in the waiting area and shorten their length of time in hospital. ${ }^{9}$ On this point, changing the list order may alter the optimum patient arrival time; we therefore recommend planning and informing the patient several days in advance to make this more achievable, as communicating this the day before or on the day of surgery can be challenging. Second, it allows supervisors to select cases which are suitable for trainees and subsequently allocate adequate time slots. ${ }^{10}$ This aspect is imperative given the detrimental impact that COVID-19 disruptions have had on cataract surgical training worldwide. $^{11}$

When asked to order the five hypothetical patients with cataract, majority of the respondents placed the least risky cases first (case A and case B), followed by cases of intermediate risk (case $\mathrm{C}$ and case $\mathrm{E}$ ), leaving the riskiest (case D) for last. Several studies have suggested that starting with the easier or less risky cases allows the surgeon to 'warm-up' and thus be more confident in managing more complex and risky cases. ${ }^{45}$ Although this trend was perceptible for the majority, there was no significant difference between the numbers of surgeons who preferred to start with the least risky compared with the most at risk case.

Due to limitations in the survey collections methods, we were unable to randomise the order in which individual participants were presented with either the case notes or the CORs. However no significant correlation was demonstrated between the responses of the participants and the default order of the case notes, the default order of the CORs or the ascending order of the CORs. This suggests that response bias secondary to non-randomisation of the question order was not an issue in this study.

Interestingly, using either the case notes only or the CORs only as the criterion to order the list did not change the preferential order. This supports the potential to use them interchangeably and thus the prospect of using CORs as a suitable criterion to order the theatre list. The reported preference for case details, rather than just the CORs, may reflect the respondents' unfamiliarity with CORs. Nevertheless, in the light of the overall positive feedback from respondents regarding a computer-automated list ordering based on the surgeon's preferred complexity order, an objective score for risk stratification, such as COR, would be necessary to enable this. In this regard, it would be worth including a score not limited to the risk of PCR, as different risk stratification systems have also been demonstrated to have the potential to reduce the rate of intraoperative complications. ${ }^{512}$

In conclusion, this survey demonstrates that cataract surgeons do prefer to choose the order of their list and that this choice is indeed influenced by the complexity of the case. Additionally, this survey supports that potential use of computer-automated list ordering based on an objective score for risk stratification, such as CORs.

Contributors KM, VR and SK conceived the idea and design for this study. KM, MF and RK contributed to the data acquisition, which were analysed by KM. Data were interpreted by KM, MF and VR. KM and MF drafted the manuscript. RK, VR and SK critically revised the draft. SK is the guarantor for this work. All authors approved the final manuscript for publication.

Funding The authors have not declared a specific grant for this research from any funding agency in the public, commercial or not-for-profit sectors. 
Competing interests None declared.

Patient consent for publication Not required.

Ethics approval The survey conformed to the Declaration of Helsinki.

Provenance and peer review Not commissioned; externally peer reviewed.

Data availability statement All data relevant to the study are included in the article or uploaded as supplementary information.

Supplemental material This content has been supplied by the author(s). It has not been vetted by BMJ Publishing Group Limited (BMJ) and may not have been peer-reviewed. Any opinions or recommendations discussed are solely those of the author(s) and are not endorsed by BMJ. BMJ disclaims all liability and responsibility arising from any reliance placed on the content. Where the content includes any translated material, BMJ does not warrant the accuracy and reliability of the translations (including but not limited to local regulations, clinical guidelines, terminology, drug names and drug dosages), and is not responsible for any error and/or omissions arising from translation and adaptation or otherwise.

Open access This is an open access article distributed in accordance with the Creative Commons Attribution Non Commercial (CC BY-NC 4.0) license, which permits others to distribute, remix, adapt, build upon this work non-commercially, and license their derivative works on different terms, provided the original work is properly cited, appropriate credit is given, any changes made indicated, and the use is non-commercial. See: http://creativecommons.org/licenses/by-nc/4.0/.

ORCID iD

Keri McLean http://orcid.org/0000-0002-8907-1176

\section{REFERENCES}

1 Zaidi FH, Corbett MC, Burton BJL, et al. Raising the benchmark for the 21 st century--the 1000 cataract operations audit and survey: outcomes, Consultant-supervised training and sourcing NHS choice. Br J Ophthalmol 2007;91:731-6.

2 Narendran N, Jaycock P, Johnston RL, et al. The Cataract National Dataset electronic multicentre audit of 55567 operations: risk stratification for posterior capsule rupture and vitreous loss. Eye 2009;23:31-7.

3 Ionides A, Minassian D, Tuft S. Visual outcome following posterior capsule rupture during cataract surgery. $\mathrm{Br} \mathrm{J}$ Ophthalmol 2001;85:222-4.

4 Ruan CW, Win SH, Francis KE, et al. Optimizing the cataract order of the list in modern surgery. $J$ Cataract Refract Surg 2014;40:842-3.

5 Pike TW, Mushtaq F, Mann RP, et al. Operating list composition and surgical performance. Br J Surg 2018;105:1061-9.

6 Abdalla G, Moran-Atkin E, Chen G, et al. The effect of warmup on surgical performance: a systematic review. Surg Endosc 2015;29:1259-69.

7 Pike TW, Pathak S, Mushtaq F, et al. A systematic examination of preoperative surgery warm-up routines. Surg Endosc 2017;31:2202-14.

8 Armstrong I. Day surgery theatre utilisation. In: Colvin JR, Peden CJ, eds. Raising the standard: a compendium of audit recipes. 3rd edn. London: The Royal College of Anaesthetists, 2012: 155-69.

9 Romano MR, Montericcio A, Montalbano C. Facing COVID-19 in ophthalmology department. Curr Eye Res 2020;23:1-6.

10 Mangan MS, Atalay E, Arıcı C, et al. Comparison of different types of complications in the phacoemulsification surgery learning curve according to number of operations performed. Tjo 2016;46:7-10.

11 Ferrara M, Romano V, Steel DH, et al. Reshaping ophthalmology training after COVID-19 pandemic. Eye 2020;34:2089-97.

12 Pooprasert P, Hansell J, Young-Zvandasara T, et al. Can applying a risk stratification system, preoperatively, reduce intraoperative complications during phacoemulsification? Curr Eye Res 2021;46:318-23. 